Appl. Phys. B 54, 295-302 (1992)

\title{
Small Volume Coaxial Discharge as Precision Testbed for OD-Models of XeCl Lasers
}

\author{
W. Bötticher, H. Lück, St. Niesner, and A. Schwabedissen \\ Institut für Plasmaphysik, Universität Hannover, W-3000 Hannover, Fed. Rep. Germany
}

Received 24 October 1991/Accepted 22 January 1992

\begin{abstract}
In order to check the predictions of 0D-models experimentally, a small coaxial discharge configuration for the generation of homogeneous high pressure glow discharges (diameter $11 \mathrm{~mm}$, length $20 \mathrm{~mm}$ ) in rare gas halogen excimer laser gas mixtures under accurately controlled conditions has been developed. It uses X-ray preionization and a special pulse-forming network (PFN) delivering fast rising ( $8 \mathrm{~ns})$ single square pulses $\left(U_{0}=25 \mathrm{kV} ; I=300 \mathrm{~A} ; \tau=100 \mathrm{~ns}\right)$. Discharge current and voltage are measured precisely by a capacitive voltage divider and a shunt integrated into the discharge chamber. All circuit data needed for the model calculations have been evaluated. Interferometric and spectroscopic diagnostics of the bulk of the discharge and of the cathode sheath have been performed. First results for $\mathrm{Ne} / \mathrm{Xe} / \mathrm{HCl}$ mixtures are compared with model calculations.
\end{abstract}

PACS: $42.55 . \mathrm{Gp}, 52.65 .+\mathrm{z}, 52.80 . \mathrm{Hc}$

The technical development of $\mathrm{XeCl}^{*}$ excimer lasers pumped by self-sustained discharges is much more advanced than the knowledge of the physics of these transient high-pressure glow discharges. There is no self-consistent model, predicting the spatial and temporal development taking into account the slightly inhomogeneous external electric field $E_{\mathrm{e}}$ due to the electrode profiles as well as the inhomogeneous preionization electron density $n_{\mathrm{eo}}(\mathbf{r})$ produced by an external VUV or X-ray preionization system. Such a selfconsistent model has to include the full reaction kinetics, the non-Maxwellian electron energy distribution function and the space charge generated electric fields $E_{\mathrm{sp}}$. The lack of such a 2D- or 3Dmodel is not only due to numerical problems and the large computing times to be expected. The up to now not very precise knowledge of the quantitative aspects of the reaction kinetics did not justify the development of true 2D-codes.

The present knowledge of the reaction kinetics of $\mathrm{XeCl}^{*}$ lasers comes from predictions of spatially homogeneous $0 \mathrm{D}$ models [1-13], which in some cases have been compared with spectroscopic and interferometric measurements from existing laser devices $[8,14-17]$. As lasers are not built to give easy access for optical plasma diagnostic methods and precise measurements of discharge voltage $U_{\mathrm{D}}(t)$ and current $I_{\mathrm{D}}(t)$, the accuracy of such comparisons is limited.

This paper presents a detailed description of a smallvolume coaxial discharge configuration especially developed to check precisely the predictions of 0D-models. First exper- imental results are compared with model calculations using an improved method to solve the Boltzmann equation [18].

The configuration has been designed to generate flattop single square-wave current pulses with current densities of $j=100-1000 \mathrm{~A} / \mathrm{cm}^{2}$ in $\mathrm{Ne} / \mathrm{Xe} / \mathrm{HCl}$ mixtures typical of $\mathrm{XeCl}^{*}$ lasers. Square current pulses allow a precise measurement of the quasi-steady-state voltage for a comparison with model predictions because inductive corrections of the measured voltages are negligible during the quasi steady-state phase. During the breakdown phase inductive corrections of the measured voltage can be performed because the discharge current is recorded simultaneously to the discharge voltage with a synchronisation of $0.5 \mathrm{~ns}$. Largediameter plane electrodes and an X-ray preionization system serve to produce very homogeneous cylindrical discharges (typically $11 \mathrm{~mm}$ diameter, $20 \mathrm{~mm}$ electrode gap). By using carefully polished and cleaned $\mathrm{Ni}$ cathodes the formation of hot-spots on the cathode surface and filaments in the bulk of the discharge can be avoided. The PFN uses a laser triggered spark gap producing a voltage pulse $U_{\mathrm{PFN}}(t)$ with a short rise time. $U_{\mathrm{PFN}}(t)$ can easily be measured. The difficulties to model the time depending conductance of the switch are thus avoided. This is of great advantage for investigations of the avalanche stage of the discharge.

Four large windows give easy access for optical diagnostics of the bulk of the discharge as well as the cathode layer. The partial pressure of $\mathrm{HCl}$ is monitored at a pre- 
cision of about $5 \%$ by infrared absorption spectroscopy of the $P(1)$ lines of $\mathrm{H}^{35} \mathrm{Cl}$ and $\mathrm{H}^{37} \mathrm{Cl}(\lambda=3.49 \mu \mathrm{m})$ [19]. Radial distributions of effective $\mathrm{Xe}^{*}$ and $\mathrm{Cl}^{*}$ number densities measured by laser absorption spectroscopy are used to judge the homogeneity of the discharge.

As the most sensitive check of the reaction kinetics used in a $0 \mathrm{D}$-model is given by the voltage $U_{\mathrm{D}}(t)$ between the electrodes special care was taken to integrate a shunt and a capacitive voltage divider into the discharge chamber. Characteristic data to be measured are the breakdown delay and the voltage $U_{\mathrm{SS}}$ during the quasi steady-state. The total current $I_{\mathrm{D}}$ is impressed due to the large value of the internal resistance $(R+Z \approx 100 \Omega)$ of the PFN. The current flowing in the observed halo will have no large effect on $U_{\mathrm{D}}(t)$ as long as the electron energy distribution function and the reaction kinetics are insensitive to minor changes of $n_{\mathrm{e}}$. The most uncertain assumption of the 0D-model is the neglect of the cathode fall. If the cathode fall is characterized by a voltage $U_{\mathrm{C}}$ and a thickness $d_{\mathrm{C}}$ (including the negative glow), one gets the electric field in the homogeneous bulk as

$E_{\mathrm{B}}=\left(U_{\mathrm{D}} / d\right)\left[\left(1-U_{\mathrm{C}} / U_{\mathrm{D}}\right) /\left(1-d_{\mathrm{C}} / d\right)\right]$,

with electrode gap $d$.

In 0D-models $E_{\mathrm{B}}=U_{\mathrm{D}} / d$ is assumed. Whereas $d_{\mathrm{C}} / d<$ $10^{-2}$ will be valid, $U_{\mathrm{C}} / U_{\mathrm{D}}$ is unknown and may be as large as 0.2 . This deviation is much larger than the error of $U_{\mathrm{D}} / d$ in our experiment. A possible way of determining $U_{\mathrm{C}}$ is to measure $U_{\mathrm{D}}$ in two discharges with different electrode gaps $d$ and otherwise unchanged parameters in order to get no change of the cathode fall and the bulk field. This procedure should be possible in our experimental arrengement.

\section{Experimental Set-Up}

\subsection{The Discharge Chamber}

In order to obtain well-defined discharge parameters a special discharge chamber, having rotational symmetry (Fig. 1), has been built. Wall materials are nickel-plated brass and PTFE in order to keep the $\mathrm{HCl}$ consumption low. The pulse transmission line of the pulse generator is connected to the cathode via a $\mathrm{CuSO}_{4}$ current limiting resistor in the form of a hollow cylinder to reduce the skin effect. For the investigation of a variety of cathode materials exchangeable electrode insets of $3 \mathrm{~cm}$ in diameter are used. The electrode gap can be changed from 5 to $20 \mathrm{~mm}$. An X-ray source behind the anode preionizes the cylindrical discharge region. The discharge cross section and thus the current density can be changed with the help of lead apertures between the X-ray source and the discharge. The central region of the anode, made of aluminium (AlCuMg1F40) is highly transparent for $\mathrm{X}$-rays. Charging the PFN to about $30 \mathrm{kV}$ causes current densities during the quasi steady-state phase of approximately $300 \mathrm{~A} / \mathrm{cm}^{2}$ if a lead aperture of $1.1 \mathrm{~cm}$ in diameter is used.

As the discharge chamber is arranged horizontally the buoyancy of the heated discharge channel produces a gas flow parallel to the electrode surfaces. This gas flow is sufficient to clear the discharge channel between two successive pulses for typical pulse repetition frequencies of $\leq 0.25 \mathrm{~Hz}$.

\subsection{The Preionization System}

To achieve a homogeneous preionization electron density of about $10^{15} / \mathrm{m}^{3}$ within the discharge volume we use a home-

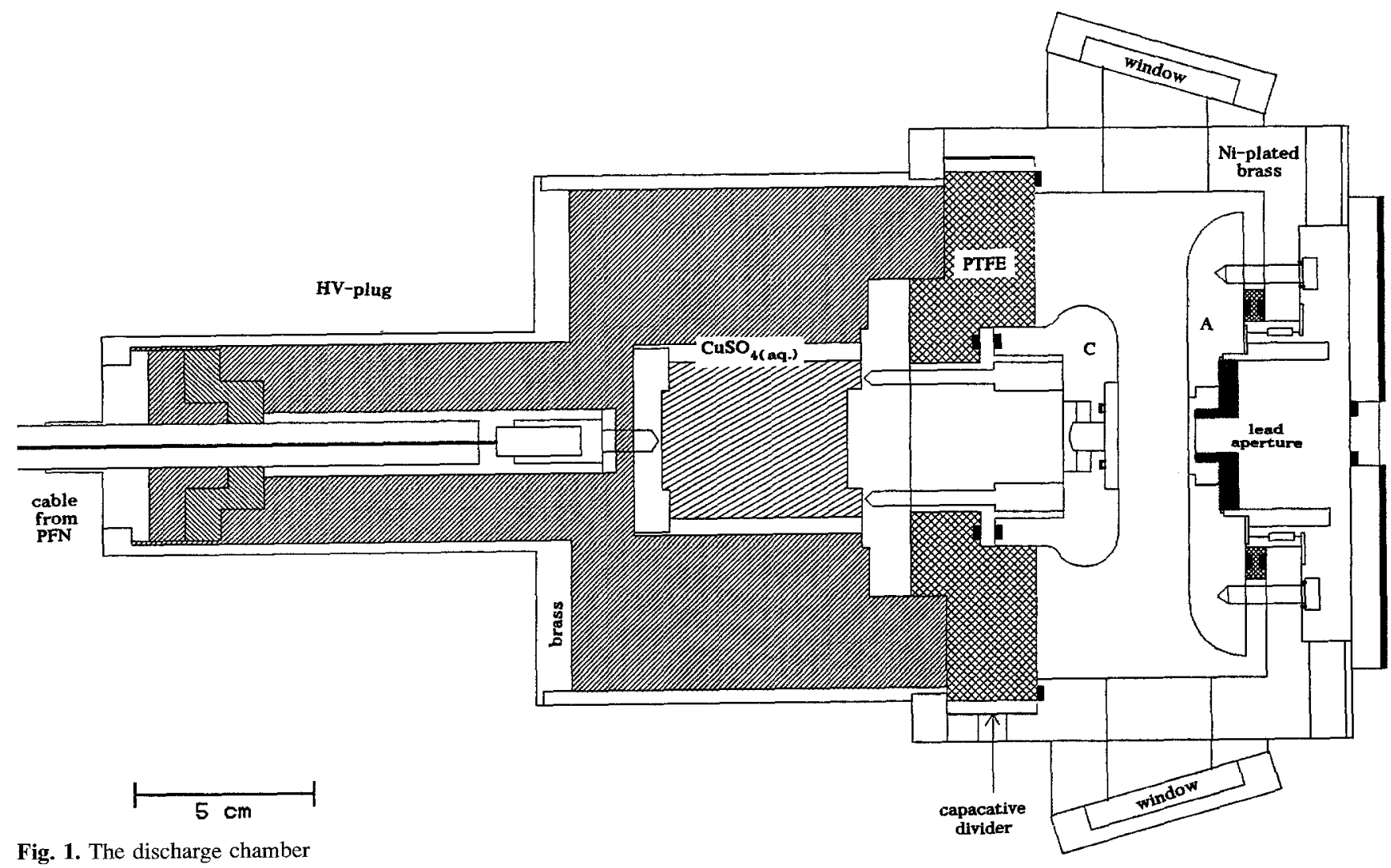




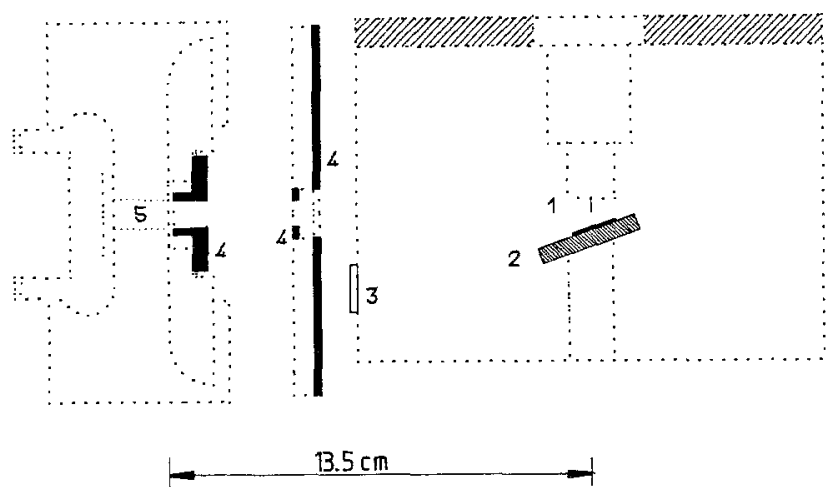

Fig. 2. Geometry of the X-ray tube and the lead apertures $l$ tantalum knife edge cathode, 2 tungsten anode (radiating area indicated), 3 plastic scintillator, 4 lead apertures, 5 discharge volume

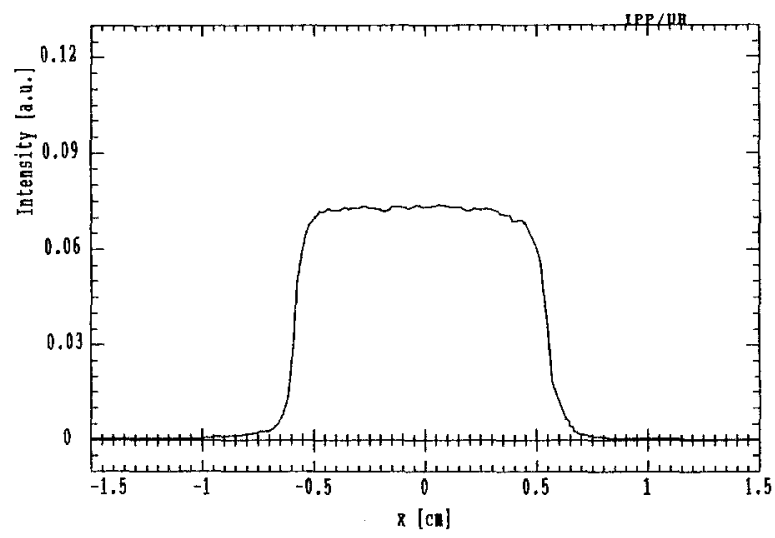

Fig. 3. Radial X-ray distribution in front of the cathode

made $\mathrm{X}$-ray tube [20, 21]. It uses a knife edge field emission cathode of tantalum ( $10 \mu \mathrm{m}$ thick, approximately $5 \mathrm{~mm}$ wide) and an anode of massive tungsten. The geometry is shown in Fig. 2. Using a pinhole camera for taking an image of the anode we found that the X-ray emitting spot has a radius of about $0.5 \mathrm{~cm}$. The intensity of the X-ray pulse and the synchronization with the discharge voltage can be monitored with the help of a scintillator in front of the X-ray tube (see Fig. 2). A pulse-forming network including an $L C$-pulse- forming line and a 1:5 pulse transformer supplies the anodecathode-gap of approximately $6 \mathrm{~mm}$ with a voltage pulse of $50 \mathrm{~ns}$ duration and a peak voltage of about $100 \mathrm{kV}$. As the PFNs of the discharge and of the X-ray tube are triggered independently, the delay between the preionization and the discharge can easily be varied. Figure 12 shows the typical synchronization between $\mathrm{X}$-rays and discharge voltage and current pulse.

The absolute preionization density can be measured by using the discharge chamber as an electron-drift-chamber. The stability of the preionization electron density of about $\pm 10 \%$ on a time scale of hours and the long-term decay of about $-30 \%$ in a period of $10^{4}-10^{5}$ shots are permanently monitored by the scintillator signal.

The distribution of the X-ray intensity in the discharge chamber is determined with an X-ray sensitive photographic film and is found to be about three orders of magnitude smaller outside the discharge channel than inside (see Fig. 3).

\subsection{The Pulse-Forming Network (PFN)}

Investigations of the footprints of the hot-spots on the cathode surface or time-integrated photographs require a single current pulse without any after-pulses. A PFN that discharges a coaxial cable will produce multiple pulses if the load does not match the impedance of the cable. If a gas discharge is part of the load, an impedance mismatch cannot be avoided during the breakdown phase of the discharge. Emission spectra taken with a spectograph and a streak camera showed that the filamentation of the discharge occurred mainly during the reflected current pulses of such a cable pulse generator. The reflections returning back to the discharge can be avoided by using a pulse generator as proposed by Proskurovsky et al. [22].

This pulse generator (see Fig. 4) produces a single square voltage pulse. The outer conductor of a coaxial cable $(Z=$ $50 \Omega, L=20 \mathrm{~m}$ ) is charged to a voltage $U_{0}$ and then discharged into the transmission line via a fast high-voltage switch. As a result square-wave pulses with an amplitude of $-U_{0} / 2$ and a duration of $100 \mathrm{~ns}$ will be sent into the transmission lines No. 1 and No. 2. The pulse travelling down line

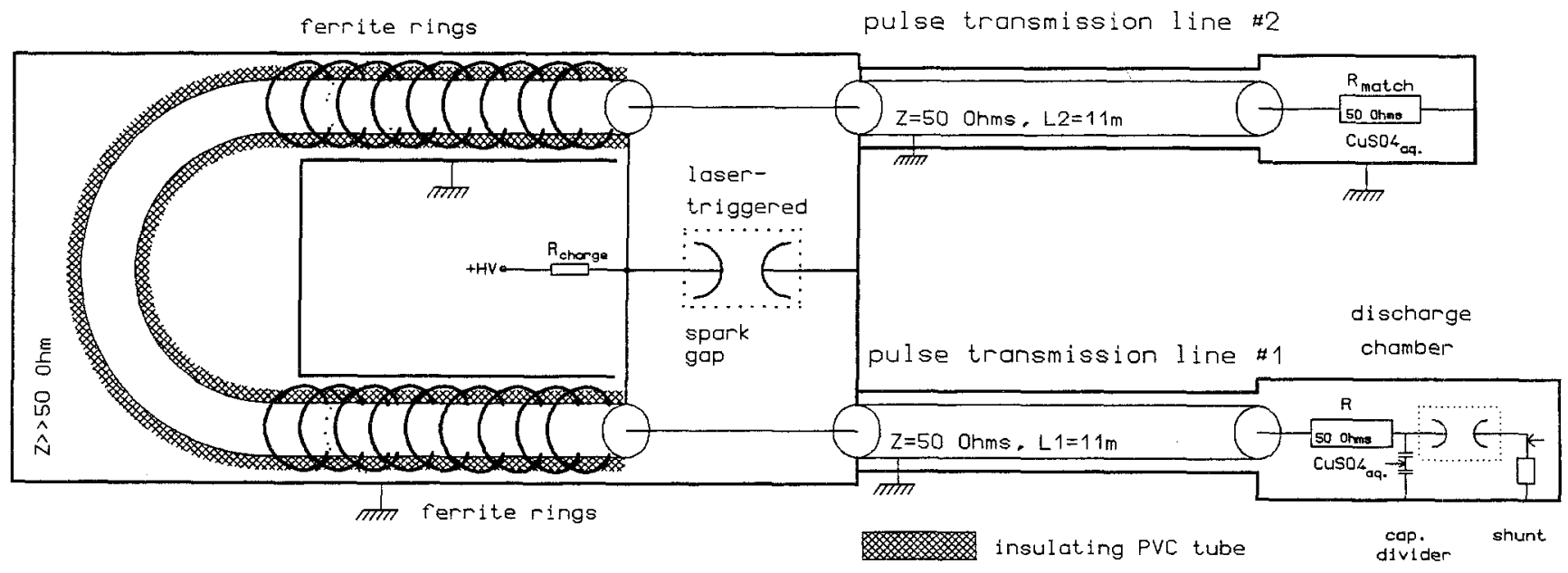

Fig. 4. Principle-sketch of the pulse generator. Pulse forming line ( $Z=50 \mathrm{Ohms}, L 3=20 \mathrm{~m}$ ) 
No. 2 will be absorbed at the matching-resistor $R_{\text {match }}=$ $50 \Omega$. If, as during the breakdown phase, the impedances of the discharge and the transmission line No. 1 are not the same, the reflected pulse will travel back the transmission line No. 1, the pulse-forming line and transmission line No. 2, be absorbed at the matching resistor at the other end of the device and will not return to the discharge. Subsequent reignitions of the discharge are thus avoided. As matching the $\mathrm{CuSO}_{4}$ resistor to the pulse transmission line is not necessary it may be altered to change the current density in the discharge.

Inductances at the spark gap, which is used as a fast highvoltage switch, cannot totally be avoided. Small parts of the pulse returning from the discharge will hence be reflected at these inductances and reappear at the discharge. If the cable length $L 1>L 3 / 2$, the reflections will reach the discharge only after the end of the main pulse and will therefore have no influence on the voltage measurement. Time-integrating techniques, like open-shutter photographs taken of the discharge, will not be affected by these reflections, because the power dissipated is small compared to the main pulse (see Fig. 5).

To reduce electromagnetic noise created by discharging of the outer conductor of the coaxial line, it is necessary to shield the pulse-forming line. The line formed by the outer conductor of the pulse-forming line and the shielding has as well to be discharged through the spark gap (see Sect. 1.3.1). A small impedance of this wave guide will

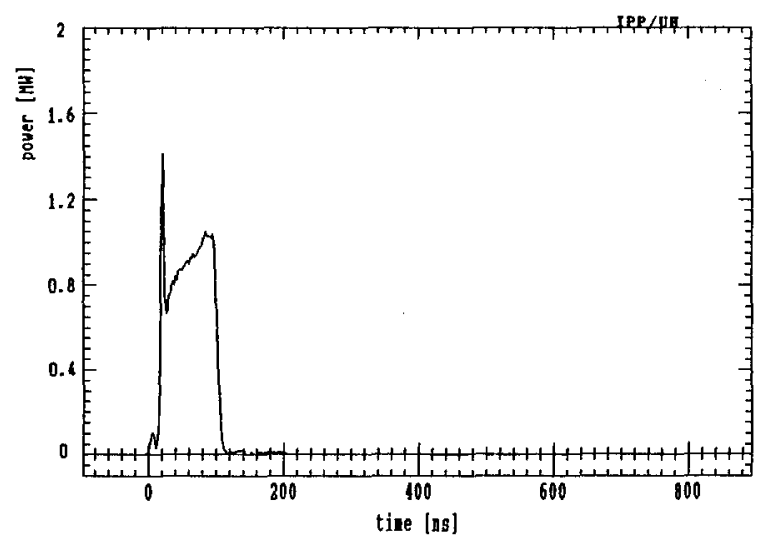

Fig. 5. Power dissipated in the discharge

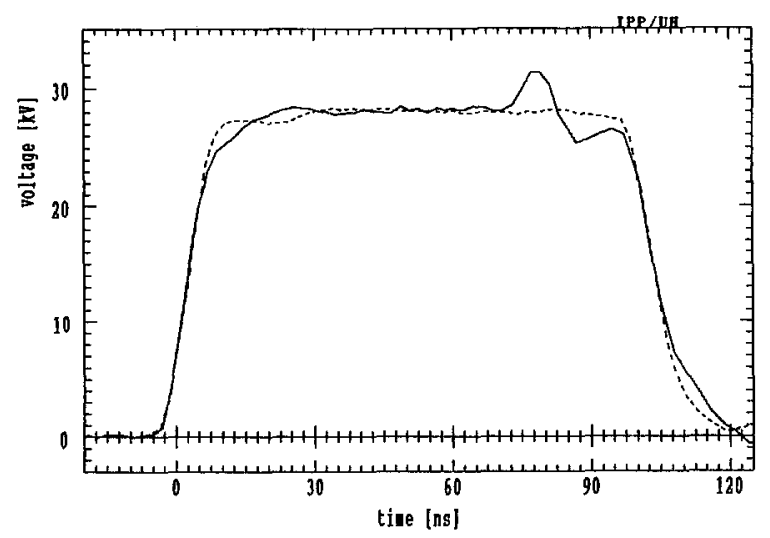

Fig. 6. Influence of the ferrite rings on the voltage $U_{\mathrm{CD}}(t)$. (-- with ferrite rings; - without ferrite rings) worsen the switching characteristics of the spark gap because of the large current to be switched. The shielding is therefore realised by spiralling the line into a metal container with sufficient space left to the wall to keep the capacitance low. Between this box and the spark gap the shielding is made from aluminium tubes with a diameter of approximately $10 \mathrm{~cm}$. The capacitance formed by the outer conductor of the line and these aluminium tubes proved to be too large to obtain a proper square pulse shape. Adding ferrite cores to the line on the whole length of the aluminium shielding $(\sim 1.5 \mathrm{~m})$ increases the inductance and suppresses the pulse distortions produced by the secondary modes on the line (see Fig. 6).

The time constant for charging the pulse-forming line according to $R_{\text {charge }}$ and $C_{\mathrm{PFL}}$ has to be large enough to avoid self-reignitions of the still conducting spark gap. A value of $R_{\text {charge }}=5 \mathrm{M} \Omega$ giving an $R C$-time constant of $10 \mathrm{~ms}$ proved to be sufficiently large.

1.3.1 The Spark Gap. The air-pressurized spark gap used as a fast high-voltage switch is triggered with a small excimer laser (EMG50, Lambda Physik, slightly modified). The beam of the excimer laser is focussed through a small hole in the cathode onto the surface of the anode by the use of a quartz lens with $5 \mathrm{~cm}$ focal length.

To obtain fast rise times, small $L C$-circuits are added close to the spark gap, which causes a small voltage overshooting at the leading edge of the pulse $U_{\mathrm{PFN}}(t)$. Varying these $L C$-circuits the best compromise of rise time and voltage overshooting could be found.

\subsection{Measurement of Voltage and Current}

1.4.I The Capacitive Voltage Divider. The capacitive divider is formed by a brass ring at the wall of the discharge chamber close to the electrode support and is insulated from the wall by a $100 \mu \mathrm{m}$ PTFE foil (see Fig. 1). This brass ring has a capacitance of $1.5 \mathrm{pF}$ to the electrode support and a capacitance of $1.2 \mathrm{nF}$ to the wall, which gives a dividingratio of $810: 1$. As the capacitances cannot be measured very accurately, the divider has to be calibrated as described below. PTFE as the insulating material inside the capacitive divider was chosen because of its chemical durability and excellent high-frequency properties. If the insulator of the electrode is made from a material having dielectric losses or a frequency-dependent permittivity, as it is the case with PVDF, severe deformations of the measured pulse occur. The voltage between the brass ring and the wall is measured by means of a fast 1:100-probe (Tektronix P6057) with $5 \mathrm{k} \Omega$ input impedance.

The place for the capacitive divider is chosen to be close enough to the discharge region to give a fairly low inductance between the divider and the discharge $\left(L_{2}=11 \mathrm{nH}\right)$ and far enough for the electrical field at the capacitive divider not to be influenced by the anode region of the discharge chamber, thus giving a constant dividing ratio independent of the electrode gap.

The signals from the voltage probe are taken to the input of a transient digitizer wave from recorder (HP 45111D) via a $50 \Omega$ cable ( $F \& G 3 \mathrm{~L} / 11)$. As the inputs of the transient digitizer do not exactly match the impedance of the cable, the length of the cable is chosen to be approximately $15 \mathrm{~m}$, 
so that pulse reflections will be delayed after the main pulse. Avoiding even minute reflections during the main pulse is essential because the voltage peak before the breakdown of the discharge is about 10 times the voltage during the quasi steady-state phase. Therefore even tiny reflections (e.g., $r=$ 1.01) will disturb the voltage measurement in the steadystate phase.

The voltage $U(t)$ to be measured and the output voltage $U_{\mathrm{CD}}(t)$ of the capacitive divider are related according to

$U(t)=U_{\mathrm{CD}}(t)+(1 / R C) \int_{0}^{t} U_{\mathrm{CD}}\left(t^{\prime}\right) d t^{\prime}$.

With $R C=6 \mu \mathrm{s}$ and $U_{\mathrm{CD}}=$ const. the correction term for a 100 ns square-wave pulse is already $1.7 \%$. As we use transient digitizer, this correction can easily be performed.

1.4.2 Calibration of the Capacitive Divider and Response to a Square Voltage Pulse. In order to calibrate the capacitive divider the open-circuit pulse of the pulse generator was measured with a 1:1000 voltage probe (Tektronix P6015) and then compared with the output of the capacitive divider. The 1:1000-probe itself was calibrated with the help of a square $200 \mathrm{~V}$ pulse with a duration of $100 \mathrm{~ns}$, which was made with a reed-relais-switched cable pulse generator capable of generating pulses up to $3 \mathrm{kV}$ with rise times $<400 \mathrm{ps}$. The dividing ratio obtained is 1:81000 including the 1:100probe of the capacitive divider. The direct application of the $200 \mathrm{~V}$ pulse to the discharge chamber gave an output pulse of

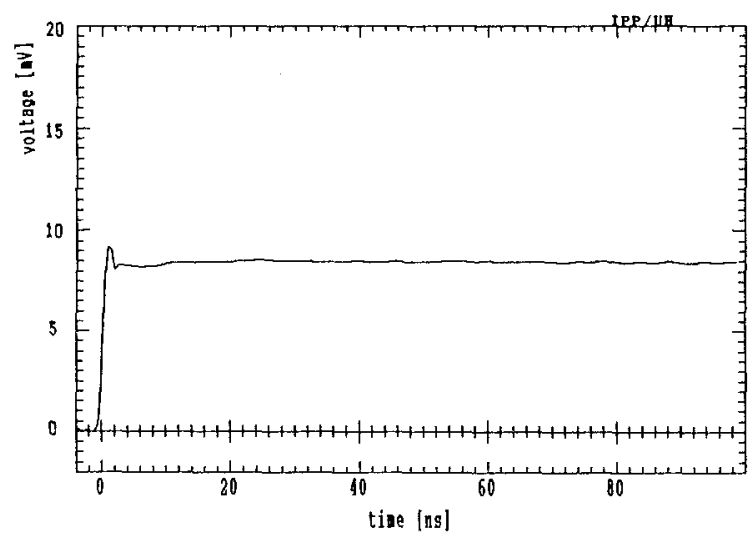

Fig. 7. Response of the capacitive divider to a step function
$2.48 \mathrm{mV}$, corresponding to a dividing ratio of $1: 80600$. The accuracy of the voltage calibration is about $2 \%$. Reflections of the voltage peak and noise during the quasi steady-state phase are $< \pm 50 \mathrm{~V}$.

The response of the capacitive divider to a step function was determined with a $3 \mathrm{kV}$ pulse of the reed-relais generator (see Fig. 7). The rise time obtained is about $1 \mathrm{~ns}$.

1.4.3 The Shunt. As indicated in Fig. 1 the current measurement is made with the help of a shunt behind the anode. It consists of a ring of $242 \Omega$ resistors in parallel, giving a total resistance of $83 \mathrm{~m} \Omega$. In order to keep the ringing in this circuit low the inductances and capacities were kept low. The resonance frequency of the shunt of $750 \mathrm{MHz}$ can be filtered out by use of an open-ended $\lambda / 4$ reactance line at the end of the $50 \Omega$ cable connecting the shunt to the transient digitizer wave form recorder, causing a short-circuit for the resonance frequency $\lambda / c$ (indicated in Fig. 8). The rise time of the whole set-up is limited to 2 ns. The resistance of the shunt incorporated into the discharge chamber was calibrated by means of a known current pulse giving a resistance of $85 \mathrm{~m} \Omega$. This value is used in the experiments. The difference of $2 \mathrm{~m} \Omega$ is assumed to be due to contact resistances.

\subsection{Equivalent Electrical Circuit of the Discharge System}

In order to perform model calculations, one has to use an equivalent electrical circuit made up of lumped elements. The circuit for the present system is shown in Fig. 8

The voltage $U_{\mathrm{PFN}}(t)$ fed into this circuit by the transmission line has been measured by the capacitive divider with a different electrode geometry to minimize stray capacitances and inductances between the cable and the capacitive divider. The measured graph of $U_{\mathrm{PFN}}^{0}(t)$ at a charging voltage of the PFN of $U_{\mathrm{CH}}^{0}=25.8 \mathrm{kV}$ is shown in Fig. 9. For other charging voltages denoted by $U_{\mathrm{CH}}, U_{\mathrm{PFN}}(t)=U_{\mathrm{PFN}}^{0}(t) U_{\mathrm{CH}} /$ $U_{\mathrm{CH}}^{0}$ is used in the model calculations.

The data given for the elements of the equivalent circuit were evaluated in an iterative procedure. Starting from values of $L, C$ taken from the geometry of the system, the response of the circuit to known square voltage pulses was calculated for short-circuited and open discharge gap. These results have been compared with voltage and current graphs measured by the capacitive divider and the shunt. $L$ 's and $C$ 's have been changed, in accordance with the limits given

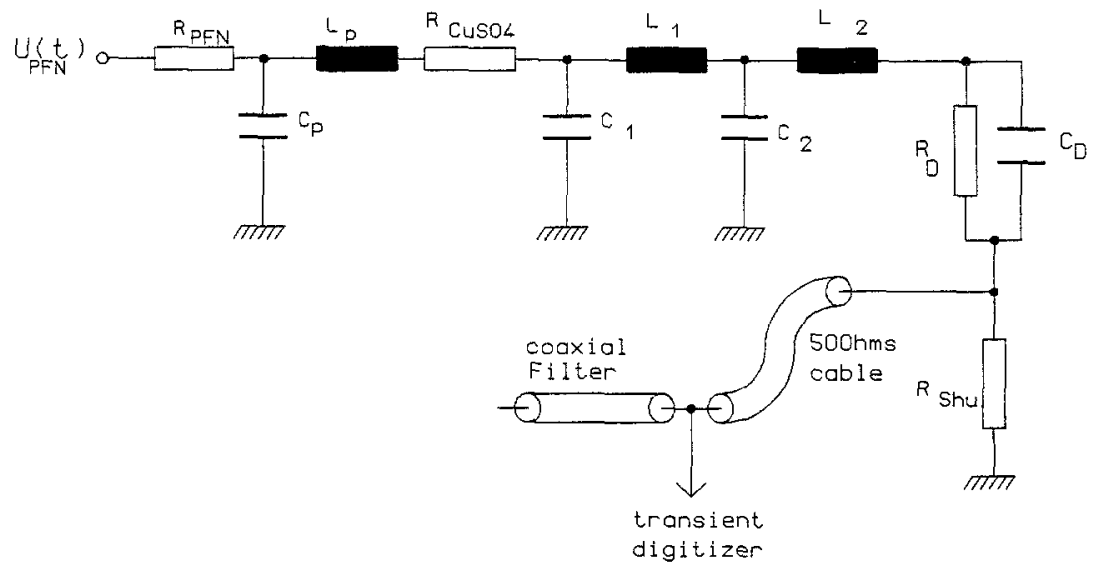

Fig. 8. The equivalent electrical circuit used in the model calculations $R_{\mathrm{PFN}}=50 \Omega ; C_{\mathrm{p}}=80 \mathrm{pF} ; L_{\mathrm{p}}=$ $70 \mathrm{nH} ; R_{\mathrm{CuSO}_{4}}=48 \Omega ; C_{1}=21 \mathrm{pF} ; L_{1}=15 \mathrm{nH}$; $C_{2}=3 \mathrm{pF} ; L_{2}=11 \mathrm{nH} ; C_{\mathrm{D}}=18.9 \mathrm{~cm} \times 1.9 \mathrm{pF} / \mathrm{d}$; $R_{\text {Shu }}=85 \mathrm{~m} \Omega ; R_{\mathrm{D}}=U_{\mathrm{D}} / I_{\mathrm{D}}$ 


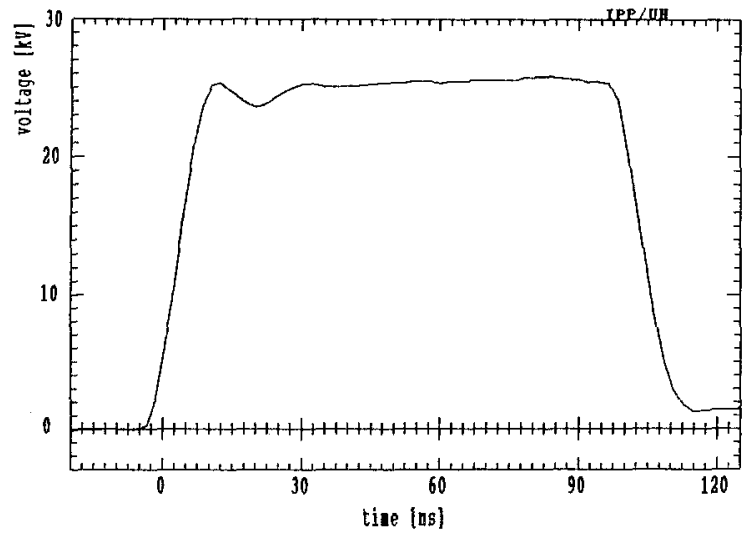

Fig. 9. The voltage $U_{\mathrm{PFN}}(t)$ delivered by the PFN at a charging voltage of $U_{\mathrm{CH}}^{0}=25.8 \mathrm{kV}$. We have defined this voltage as $U_{\mathrm{PFN}}^{0}(t)$. It is used as an input look-up-table in the model calculations

by the geometry of the conducting surfaces, until the calculations on the basis of the circuit equations reproduced the measured data.

\section{Experimental Results}

\subsection{Discharge Homogeneity}

Open-shutter colour video pictures show a homogeneous blue discharge channel of $11 \mathrm{~mm}$ diameter. We have never seen filaments in discharges with the parameters: $I_{\mathrm{D}} \sim 300 \mathrm{~A}$; $p=3$ bar; $p_{\text {xenon }}=25-100 \mathrm{mbar} ; p_{\mathrm{HCl}} \leq 7.5 \mathrm{mbar} ; d=$ $18.9 \mathrm{~mm}$. Very bright hot-spots on the cathode surface are found regularly. Only extremly carefully polished $\mathrm{Ni}$ or $\mathrm{Cu}$ cathodes develop no spots. Hot-spots on the anode have never been observed. It is believed, that all reports on anode spots are due to undetected current reversals of afterpulses. On the top of the cathode hot-spots a thin $(100 \mu \mathrm{m}$ diameter), short $(\leq 500 \mu \mathrm{m}$ length) and very bright cylindrical discharge channel is found connecting the hot-spot with the homogeneous bulk.

Schlieren pictures with $3 \mathrm{~ns}$ exposure time [23] show weak shock waves expanding radially from these plasma channels. This indicates a very strongly enhanced current density in these channels. Further investigations have to be done to show whether these channels can form the roots of filament formation due to $\mathrm{HCl}$ depletion.

Schlieren pictures and interferograms also show a weak plane shock wave leaving the cathode. To our surprise this plane cathode shock wave is not only seen inside the main discharge channel but also in the $\mathrm{Xe}^{*}$ halo (see below) found by absorption spectroscopy. The origin of this shock wave is an open question. It is assumed that gas desorption plays an important role.

The effective number densities of the XeI-transition $6 s(1 / 2)_{2}-6 p(3 / 2)_{2}, \lambda=823.39 \mathrm{~nm}$ and the CII-transition $4 s^{4} P_{5 / 2}-4 p^{4} D_{7 / 2}, \lambda=837.59 \mathrm{~nm}$ have been measured by time-resolved laser absorption spectroscopy. This technique, described in detail in [8] was improved by using a transient digitizer HP $54111 \mathrm{D}$. This made it possible, by recording the mean values of up to 40 discharges, to evaluate very small absorption signals $I / I_{0}=\exp [-T(t)]$. Optical thicknesses down to $T=0.005$ could be measured.

A comparison of lateral scans of $T(y, t)$ for some times $t_{i}$ with model calculations of $T(y)$ for a radially homogeneous discharge show good agreement for the ClI-line. Assuming a homogeneous discharge the absorption path length of the diameter of the discharge $(11 \mathrm{~mm})$ was used to derive $N_{\text {eff }}(t)$. The linear rise during the quasi steady-state of the discharge (see Fig. 10) is due to the constant production rate of $\mathrm{Cl}^{-}$ions due to dissociative attachment (electron density $N_{\mathrm{e}} \approx$ const $\approx 10^{21} \mathrm{~m}^{-3}$ ). After that the $\mathrm{Cl}^{-}$ions are converted into $\mathrm{Cl}$ atoms. The unexpected observation that the final $\mathrm{Cl}^{*}$ density is independent of the $\mathrm{HCl}$ partial pressure $\left(2.5 \mathrm{mbar}<p_{\mathrm{HCl}}<10.0 \mathrm{mbar}\right)$ is in contradiction with preliminary model calculations. Further experimental (absorption of the Hydrogen line $\mathrm{H}_{\alpha}$ ) and theoretical work is under way.

In contrast to the $\mathrm{CII}$ absorption $\mathrm{XeI}$ absorption is found not only in the main discharge channel but also in a surrounding halo. Similar halos have been found before $[8,16]$. As the outer diameter of the halo is larger than the widths of the present windows $(20 \mathrm{~mm})$, it was not possible to determine $N_{\text {eff }}\left(\mathrm{Xe}^{*}\right)$ by an Abel inversion. Lateral scans are shown in Fig. 11.

The origin of this halo in our configuration is still a puzzle. The very low X-ray dose measured outside the main

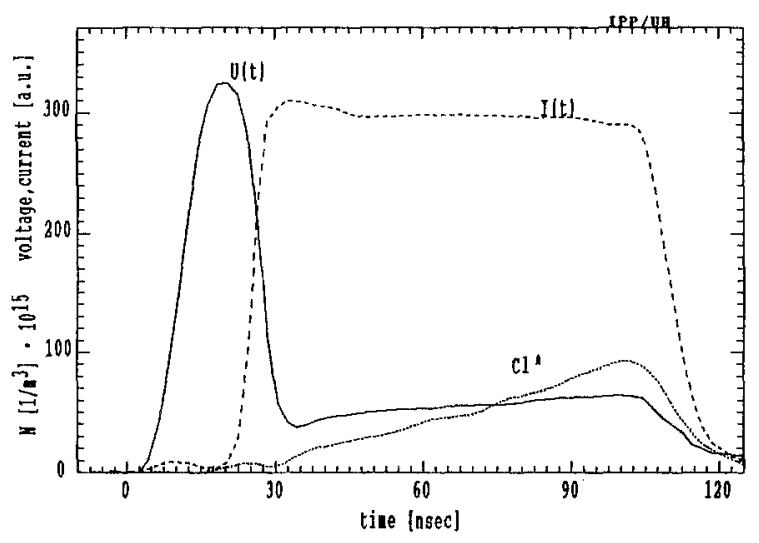

Fig. 10. Temporal development of the effective particle density of excited $\mathrm{Cl}$ atoms

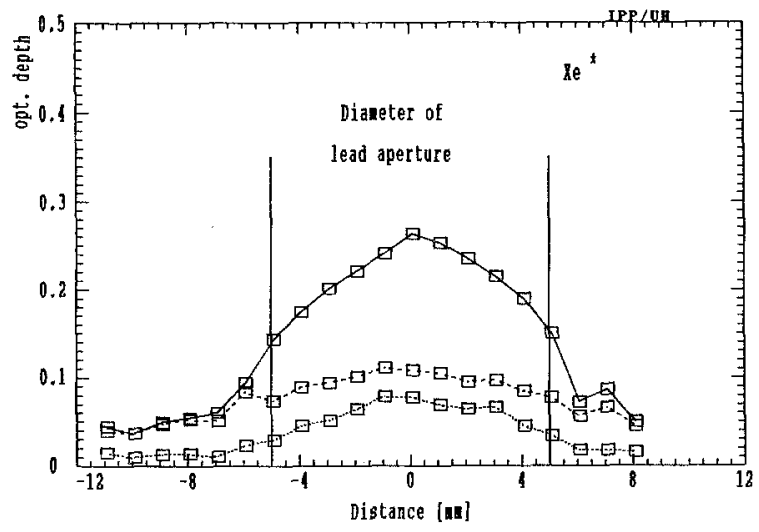

Fig. 11. Optical depth of the XeI-line as a function of the distance from the discharge center for different times $(-20 \mathrm{~ns} ;---50 \mathrm{~ns} ; \cdots$ $100 \mathrm{~ns})$ 
channel (see Fig. 3) does not produce a preionization electron density large enough to develop into a halo discharge having the measured XeI optical depths. This has been checked by preliminary model calculations in which our full OD-model was used in a parallel resistor model. The fact of some current flowing in the halo is indicated by the observed cathode shock wave. More detailed experimental and theoretical investigations are needed to clarify the situation. It may be possible that a delayed halo preionization is caused by VUV radiation emitted from the very first stages of the developing main discharge. An accurate knowledge of the division of the discharge current into the halo current and the main discharge current is crucial for precise checks of OD-models.

\subsection{Current and Voltage Measurements}

Measured data for our standard gas mixture of $\mathrm{HCl}: 5 \mathrm{mbar}$, Xe: $75 \mathrm{mbar}$, Ne: $2920 \mathrm{mbar}$ with $U_{\mathrm{CH}}=25.8 \mathrm{kV}$ and $18.9 \mathrm{~mm}$ electrode gap are shown in Fig. 12. The current flowing before the discharge breakdown is due to the electrode capacitance. First results of model calculations using our 0D-model [24] are given in Fig. 13.

The quasi steady-state voltages are reproduced accurately. The calculated breakdown delay time is too short. Even drastic changes of the HCL attachment kinetics or the preionization electron density assumed in the model calcu-

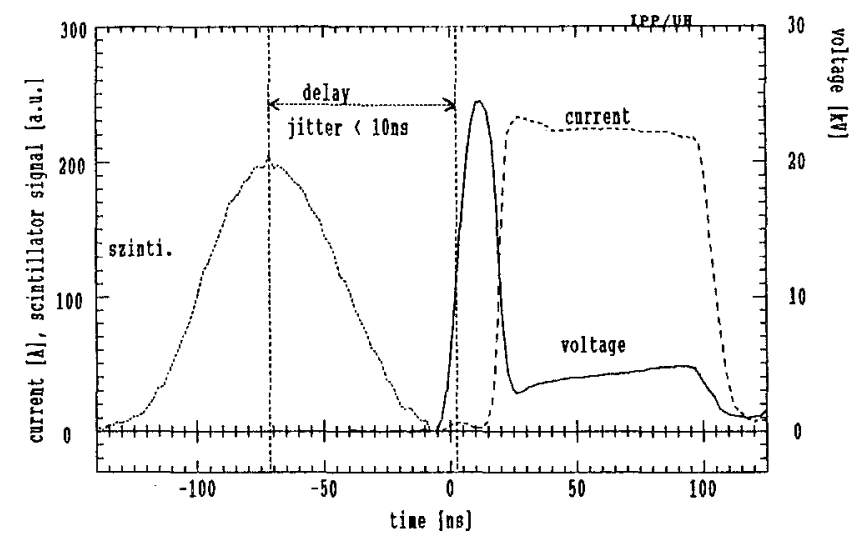

Fig. 12. Measured data of discharge voltage and current, at standard conditions (see text) and synchronization with X-ray pulse

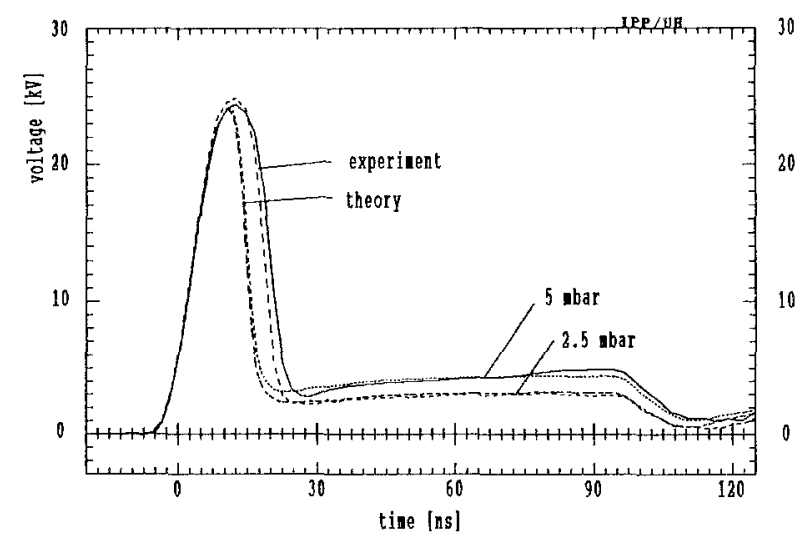

Fig. 13. Comparison of voltage graphs from the experiment and the model for standard conditions (see text) and reduced HCl pressure of $2.5 \mathrm{mbar}$ lations did not resolve this discrepancy. It may be necessary to include an assumed voltage drop $U_{\mathrm{C}}(t)$ in the cathode fall into the $0 \mathrm{D}$-model, i.e., to calculate the electric field $E_{\mathrm{B}}$ in the bulk according to

$$
E_{\mathrm{B}}(t)=\left[U_{\mathrm{D}}(t)-U_{\mathrm{C}}(t)\right] / d .
$$

\section{Conclusion}

The experimental set-up presented produces very well defined homogeneous high-pressure glow discharges. It is well suited to do spectroscopic and interferometric measurements of particle densities simultaneously with precise measurements of discharge voltage and current. As the circuit data of the PFN are accurately known the discharge can be used to check the predictions of $\mathrm{XeCl}$ and $\mathrm{KrF}$ laser models in a large parameter field.

As the spatial distribution and the degree of the preionization as well as the discharge current density can easily be changed it is possible to produce discharges showing filamentation under controlled conditions. Due to the compact design optical investigations with high spatial resolution can easily be performed.

It has been demonstrated [23] that it is possible to study the instabilities of the cathode sheath resulting in hot-spots and roots of filaments. Additionally the plane gasdynamic shock wave leaving the cathode has been visualized. Models of the temporal development of the cathode sheath in $\mathrm{XeCl}$ mixtures presently under development [25] should therefore include changes in the gas density. The importance of this effect has been pointed out for low pressure glow discharges in $[26,27]$.

Acknowledgements. This work is part of a larger project investigating the gas discharge physics of excimer lasers, which is supported by the Bundesminister für Forschung und Technologie (FE Vorhaben 13 N5406/9 and 13N5722/3), the Deutsche Forschungsgemeinschaft (Bo496/11; 12 and 13) and a grant by Asea Brown Boveri Corporate Research, Baden.

The authors are grateful to D. Backhaus who did the model calculations and to $\mathrm{M}$. Bähr who was responsible for the development of the $\mathrm{X}$-ray pulser.

\section{References}

1. A.E. Greene, C.A. Brau: IEEE J. QE-14, 951-957 (1978)

2. T.H. Johnson, L.J. Palumbo, A.M. Hunter: IEEE J. QE-15, 289 301 (1979)

3. M. Maeda, A. Takahashi, T. Mizunami, Y. Miyazoe: Jpn. J. Appl. Phys. 21 1161-1169 (1982)

4. H. Hokazono, K. Midorikawa, M. Obara, T. Fujioka: J. Appl. Phys. 56 680-690 (1984)

5. A.V. Dem'yanov, I.V. Kochetov, A.P. Narpatovich, A.N. Starostin, M.D. Taran: Sov. J. Quant. Electron. 15, 1147-1149 (1985)

6. M. Ohwa, M. Obara: J. Appl. Phys. 59, 32-41 (1986)

7. G. Stielow, Th. Hammer, W. Bötticher: Appl. Phys. B 47, 333-342 (1988)

8. Th. Hammer, W. Bötticher: Appl. Phys. B 48, 73-84 (1989)

9. V. Mihkelsoo, P. Miidla, A. Sherman, R. Sorkina, E. Tamme, A. Treshchalov: J. Phys. B: At. Mol. Opt. Phys. 22, 1489-1504 (1989)

10. C. Gorse: In Nonequilibrium Processes in Partially Ionized Gases, ed. by M. Capitelli, J. Bardsley (Plenum, New York 1990) pp. 441 
12. C. Gorse, M. Capitelli, A. Dipace: J. Appl. Phys. 67, 1118-1120 (1990)

13. E. Estocq, J. Bretagne: Proc. ICPIG, 1188-1189 (1991)

14. M.M. Turner, P.W. Smith: IEEE Trans. PS 19, 352-360 (1991)

15. A.V. Dem'yanov, V.S. Egorov, I.V. Kochetov, A.P. Narpatovich, A.A. Pastor, N.P. Penkin, P.Yu. Serdobintsev, N.N. Shubin: Sov. Quant. Electron. 16, 817-820 (1986)

16. V.É. Peét, A.B. Treshalov: Sov. Quant. Electron. 15, 1613-16119 (1985)

17. S. Choroba, W. Bötticher: Appl. Phys. B 51, 379-385 (1990)

18. R. Winkler, M.W. Wuttke: Appl. Phys. B (in press)

19. G. Schröder: Diploma Thesis, Universität Hannover (1991)
20. M. Bähr: Ph. D. Thesis, Univerität Hannover (1989)

21. W. Stieler: Diploma Thesis, Universität Hannover (1989)

22. D.I. Proskurovskii, E.B. Yankelevich: Pribory i Tekhnika Éksperimenta 5, 108-111 (1973)

23. V. Skwarczynski: Diploma Thesis, Universität Hannover (1990)

24. D. Backhaus: To be published

25. J.P. Boeuf: Private communication

26. F. Bastien, Wu Jiang Hua, P. Goguillon, E. Marode: J. Phys. D 23, 813-822 (1990)

27. E.A. Den Hartog, D.A. Doughty, J.E. Lawler: Phys. Rev. A 38, 2471-2491 (1988) 